\title{
Epsilon Numbers and Cantor Normal Form
}

\author{
Grzegorz Bancerek \\ Białystok Technical University \\ Poland
}

Summary. An epsilon number is a transfinite number which is a fixed point of an exponential map: $\omega^{\varepsilon}=\varepsilon$. The formalization of the concept is done with use of the tetration of ordinals (Knuth's arrow notation, $\uparrow \uparrow$ ). Namely, the ordinal indexing of epsilon numbers is defined as follows:

$$
\varepsilon_{0}=\omega \uparrow \uparrow \omega, \quad \varepsilon_{\alpha+1}=\varepsilon_{\alpha} \uparrow \uparrow \omega,
$$

and for limit ordinal $\lambda$ :

$$
\varepsilon_{\lambda}=\lim _{\alpha<\lambda} \varepsilon_{\alpha}=\bigcup_{\alpha<\lambda} \varepsilon_{\alpha}
$$

Tetration stabilizes at $\omega$ :

$$
\alpha \uparrow \uparrow \beta=\alpha \uparrow \uparrow \omega \text { for } \quad \alpha \neq 0 \text { and } \beta \geq \omega .
$$

Every ordinal number $\alpha$ can be uniquely written as

$$
n_{1} \omega^{\beta_{1}}+n_{2} \omega^{\beta_{2}}+\cdots+n_{k} \omega^{\beta_{k}}
$$

where $k$ is a natural number, $n_{1}, n_{2}, \ldots, n_{k}$ are positive integers, and $\beta_{1}>\beta_{2}>$ $\ldots>\beta_{k}$ are ordinal numbers $\left(\beta_{k}=0\right)$. This decomposition of $\alpha$ is called the Cantor Normal Form of $\alpha$.

MML identifier: ORDINAL5, version: $7.11 .04 \quad 4.130 .1076$

The notation and terminology used here are introduced in the following papers: $[9],[3],[8],[7],[1],[5],[6],[4],[2]$, and [10]. 


\section{Preliminaries}

For simplicity, we follow the rules: $\alpha, \beta, \gamma$ denote ordinal numbers, $m, n$ denote natural numbers, $f$ denotes a sequence of ordinal numbers, and $x$ denotes a set.

One can prove the following proposition

(1) If $\alpha \subseteq \operatorname{succ} \beta$, then $\alpha \subseteq \beta$ or $\alpha=\operatorname{succ} \beta$.

Let us note that $\omega$ is limit ordinal and every empty set is ordinal yielding.

One can verify that there exists a transfinite sequence which is non empty and finite.

Let $f$ be a transfinite sequence and let $g$ be a non empty transfinite sequence. One can check that $f^{\frown} g$ is non empty and $g^{\frown} f$ is non empty.

In the sequel $\psi, \psi_{1}, \psi_{2}$ denote transfinite sequences.

One can prove the following three propositions:

(2) If $\operatorname{dom} \psi=\alpha+\beta$, then there exist $\psi_{1}, \psi_{2}$ such that $\psi=\psi_{1} \frown \psi_{2}$ and $\operatorname{dom} \psi_{1}=\alpha$ and $\operatorname{dom} \psi_{2}=\beta$.

(3) $\operatorname{rng} \psi_{1} \subseteq \operatorname{rng}\left(\psi_{1} \frown \psi_{2}\right)$ and $\operatorname{rng} \psi_{2} \subseteq \operatorname{rng}\left(\psi_{1} \frown \psi_{2}\right)$.

(4) If $\psi_{1} \frown \psi_{2}$ is ordinal yielding, then $\psi_{1}$ is ordinal yielding and $\psi_{2}$ is ordinal yielding.

Let $f$ be a transfinite sequence. We say that $f$ is decreasing if and only if:

(Def. 1) For all $\alpha, \beta$ such that $\alpha \in \beta$ and $\beta \in \operatorname{dom} f$ holds $f(\beta) \in f(\alpha)$.

We say that $f$ is non-decreasing if and only if:

(Def. 2) For all $\alpha, \beta$ such that $\alpha \in \beta$ and $\beta \in \operatorname{dom} f$ holds $f(\alpha) \subseteq f(\beta)$.

We say that $f$ is non-increasing if and only if:

(Def. 3) For all $\alpha, \beta$ such that $\alpha \in \beta$ and $\beta \in \operatorname{dom} f$ holds $f(\beta) \subseteq f(\alpha)$.

Let us observe that every sequence of ordinal numbers which is increasing is also non-decreasing and every sequence of ordinal numbers which is decreasing is also non-increasing.

We now state the proposition

(5) For every transfinite sequence $f$ holds $f$ is infinite iff $\omega \subseteq \operatorname{dom} f$.

Let us note that every transfinite sequence which is decreasing is also finite and every sequence of ordinal numbers which is empty is also decreasing and increasing.

Let us consider $\alpha$. Observe that $\langle\alpha\rangle$ is ordinal yielding.

Let us consider $\alpha$. One can check that $\langle\alpha\rangle$ is decreasing and increasing.

Let us observe that there exists a sequence of ordinal numbers which is decreasing, increasing, non-decreasing, non-increasing, finite, and non empty.

The following propositions are true:

(6) For every non-decreasing sequence $f$ of ordinal numbers such that $\operatorname{dom} f$ is non empty holds $\bigcup f$ is the limit of $f$. 
(7) If $\alpha \in \beta$, then $n \cdot \omega^{\alpha} \in \omega^{\beta}$.

(8) If $0 \in \alpha$ and for every $\beta$ such that $\beta \in \operatorname{dom} f$ holds $f(\beta)=\alpha^{\beta}$, then $f$ is non-decreasing.

(9) If $\alpha$ is a limit ordinal number and $0 \in \beta$, then $\alpha^{\beta}$ is a limit ordinal number.

(10) If $1 \in \alpha$ and for every $\beta$ such that $\beta \in \operatorname{dom} f$ holds $f(\beta)=\alpha^{\beta}$, then $f$ is increasing.

(11) If $0 \in \alpha$ and $\beta$ is a non empty limit ordinal number, then $x \in \alpha^{\beta}$ iff there exists $\gamma$ such that $\gamma \in \beta$ and $x \in \alpha^{\gamma}$.

(12) If $0 \in \alpha$ and $\alpha^{\beta} \in \alpha^{\gamma}$, then $\beta \in \gamma$.

\section{Tetration (Knuth's Arrow Notation) of Ordinals ${ }^{1}$}

Let $\alpha, \beta$ be ordinal numbers. The functor $\alpha \uparrow \uparrow \beta$ yields an ordinal number and is defined by the condition (Def. 4).

(Def. 4) There exists a sequence $\varphi$ of ordinal numbers such that

(i) $\quad \alpha \uparrow \uparrow \beta=$ last $\varphi$,

(ii) $\operatorname{dom} \varphi=\operatorname{succ} \beta$,

(iii) $\varphi(\emptyset)=1$,

(iv) for every ordinal number $\gamma$ such that $\operatorname{succ} \gamma \in \operatorname{succ} \beta$ holds $\varphi(\operatorname{succ} \gamma)=$ $\alpha^{\varphi(\gamma)}$, and

(v) for every ordinal number $\gamma$ such that $\gamma \in \operatorname{succ} \beta$ and $\gamma \neq \emptyset$ and $\gamma$ is a limit ordinal number holds $\varphi(\gamma)=\lim (\varphi\lceil\gamma)$.

We now state a number of propositions:

(13) $\alpha \uparrow \uparrow 0=1$.

(14) $\alpha \uparrow \uparrow \operatorname{succ} \beta=\alpha^{\alpha \uparrow \uparrow \beta}$.

(15) Suppose $\beta \neq \emptyset$ and $\beta$ is a limit ordinal number. Let $\varphi$ be a sequence of ordinal numbers. If $\operatorname{dom} \varphi=\beta$ and for every $\gamma$ such that $\gamma \in \beta$ holds $\varphi(\gamma)=\alpha \uparrow \uparrow \gamma$, then $\alpha \uparrow \uparrow \beta=\lim \varphi$.

(16) $\alpha \uparrow \uparrow 1=\alpha$.

(17) $1 \uparrow \uparrow \alpha=1$.

(18) $\alpha \uparrow \uparrow 2=\alpha^{\alpha}$.

(19) $\alpha \uparrow \uparrow 3=\alpha^{\alpha^{\alpha}}$.

(20) For every natural number $n$ holds $0 \uparrow \uparrow(2 \cdot n)=1$ and $0 \uparrow \uparrow(2 \cdot n+1)=0$.

(21) If $\alpha \subseteq \beta$ and $0 \in \gamma$, then $\gamma \uparrow \uparrow \alpha \subseteq \gamma \uparrow \uparrow \beta$.

\footnotetext{
${ }^{1}$ Important fact (32)
}

$$
\alpha \uparrow \uparrow \beta=\alpha \uparrow \uparrow \omega \quad \text { for } \beta \geq \omega \text { and } \alpha>0
$$


(22) If $0 \in \alpha$ and for every $\beta$ such that $\beta \in \operatorname{dom} f$ holds $f(\beta)=\alpha \uparrow \uparrow \beta$, then $f$ is non-decreasing.

(23) If $0 \in \alpha$ and $0 \in \beta$, then $\alpha \subseteq \alpha \uparrow \uparrow \beta$.

(24) If $1 \in \alpha$ and $m<n$, then $\alpha \uparrow \uparrow m \in \alpha \uparrow \uparrow n$.

(25) If $1 \in \alpha$ and $\operatorname{dom} f \subseteq \omega$ and for every $\beta$ such that $\beta \in \operatorname{dom} f$ holds $f(\beta)=\alpha \uparrow \uparrow \beta$, then $f$ is increasing.

(26) If $1 \in \alpha$ and $1 \in \beta$, then $\alpha \in \alpha \uparrow \uparrow \beta$.

(27) For all natural numbers $n, k$ holds $n^{k}=n^{k}$.

Let $n, k$ be natural numbers. Observe that $n^{k}$ is natural.

Let $n, k$ be natural numbers. One can check that $n \uparrow \uparrow k$ is natural.

Next we state several propositions:

(28) For all natural numbers $n, k$ such that $n>1$ holds $n \uparrow \uparrow k>k$.

(29) For every natural number $n$ such that $n>1$ holds $n \uparrow \uparrow \omega=\omega$.

(30) If $1 \in \alpha$, then $\alpha \uparrow \uparrow \omega$ is a limit ordinal number.

(31) If $0 \in \alpha$, then $\alpha^{\alpha \uparrow \uparrow}=\alpha \uparrow \uparrow \omega$.

(32) If $0 \in \alpha$ and $\omega \subseteq \beta$, then $\alpha \uparrow \uparrow \beta=\alpha \uparrow \uparrow \omega$.

\section{CRitical Numbers ${ }^{2}$}

In this article we present several logical schemes. The scheme CriticalNumber2 deals with an ordinal number $\mathcal{A}$, a sequence $\mathcal{B}$ of ordinal numbers, and a unary functor $\mathcal{F}$ yielding an ordinal number, and states that:

$\mathcal{A} \subseteq \cup \mathcal{B}$ and $\mathcal{F}(\cup \mathcal{B})=\cup \mathcal{B}$ and for every $\beta$ such that $\mathcal{A} \subseteq \beta$ and $\mathcal{F}(\beta)=\beta$ holds $\cup \mathcal{B} \subseteq \beta$

provided the following requirements are met:

- For all $\alpha, \beta$ such that $\alpha \in \beta$ holds $\mathcal{F}(\alpha) \in \mathcal{F}(\beta)$,

- Let given $\alpha$. Suppose $\alpha$ is a non empty limit ordinal number. Let $\varphi$ be a sequence of ordinal numbers. If $\operatorname{dom} \varphi=\alpha$ and for every $\beta$ such that $\beta \in \alpha$ holds $\varphi(\beta)=\mathcal{F}(\beta)$, then $\mathcal{F}(\alpha)$ is the limit of $\varphi$,

- $\operatorname{dom} \mathcal{B}=\omega$ and $\mathcal{B}(0)=\mathcal{A}$, and

- For every $\alpha$ such that $\alpha \in \omega$ holds $\mathcal{B}(\operatorname{succ} \alpha)=\mathcal{F}(\mathcal{B}(\alpha))$.

The scheme CriticalNumber3 deals with an ordinal number $\mathcal{A}$ and a unary functor $\mathcal{F}$ yielding an ordinal number, and states that:

There exists $\alpha$ such that $\mathcal{A} \in \alpha$ and $\mathcal{F}(\alpha)=\alpha$ provided the following requirements are met:

- For all $\alpha, \beta$ such that $\alpha \in \beta$ holds $\mathcal{F}(\alpha) \in \mathcal{F}(\beta)$, and

\footnotetext{
${ }^{2} \mathcal{F}$ is increasing continuous map of ordinals and $\alpha=\mathcal{F}(\alpha)$ is a critical number of $\mathcal{F}$
} 
- Let given $\alpha$. Suppose $\alpha$ is a non empty limit ordinal number. Let $\varphi$ be a sequence of ordinal numbers. If $\operatorname{dom} \varphi=\alpha$ and for every $\beta$ such that $\beta \in \alpha$ holds $\varphi(\beta)=\mathcal{F}(\beta)$, then $\mathcal{F}(\alpha)$ is the limit of $\varphi$.

\section{EPSILON NUMBERS ${ }^{3}$}

Let $\alpha$ be an ordinal number. We say that $\alpha$ is epsilon if and only if:

(Def. 5) $\quad \omega^{\alpha}=\alpha$.

One can prove the following proposition

(33) There exists $\beta$ such that $\alpha \in \beta$ and $\beta$ is epsilon.

Let us note that there exists an ordinal number which is epsilon.

Let $\alpha$ be an ordinal number. The first $\varepsilon$ greater than $\alpha$ yielding an epsilon number is defined by the conditions (Def. 6).

(Def. 6)(i) $\quad \alpha \in$ the first $\varepsilon$ greater than $\alpha$, and

(ii) for every epsilon number $\beta$ such that $\alpha \in \beta$ holds the first $\varepsilon$ greater than $\alpha \subseteq \beta$.

One can prove the following four propositions:

(34) If $\alpha \subseteq \beta$, then the first $\varepsilon$ greater than $\alpha \subseteq$ the first $\varepsilon$ greater than $\beta$.

(35) Suppose $\alpha \in \beta$ and $\beta \in$ the first $\varepsilon$ greater than $\alpha$. Then the first $\varepsilon$ greater than $\beta=$ the first $\varepsilon$ greater than $\alpha$.

(36) The first $\varepsilon$ greater than $0=\omega \uparrow \uparrow \omega$.

(37) For every epsilon number $e$ holds $\omega \in e$.

One can check that every ordinal number which is epsilon is also non empty limit ordinal.

One can prove the following propositions:

(38) For every epsilon number $e$ holds $\omega^{e^{\omega}}=e^{e^{\omega}}$.

(39) For every epsilon number $e$ such that $0 \in n$ holds $e \uparrow \uparrow(n+2)=\omega^{e \uparrow \uparrow(n+1)}$.

(40) For every epsilon number $e$ holds the first $\varepsilon$ greater than $e=e \uparrow \uparrow \omega$.

Let $\alpha$ be an ordinal number. The functor $\varepsilon_{\alpha}$ yields an ordinal number and is defined by the condition (Def. 7).

(Def. 7) There exists a sequence $\varphi$ of ordinal numbers such that

(i) $\varepsilon_{\alpha}=$ last $\varphi$,

(ii) $\operatorname{dom} \varphi=\operatorname{succ} \alpha$,

(iii) $\varphi(\emptyset)=\omega \uparrow \uparrow \omega$,

(iv) for every ordinal number $\beta$ such that $\operatorname{succ} \beta \in \operatorname{succ} \alpha$ holds $\varphi(\operatorname{succ} \beta)=$ $\varphi(\beta) \uparrow \uparrow \omega$, and

\footnotetext{
${ }^{3}$ An ordinal number $\alpha$ is epsilon iff it is a critical number of expnential map: $\alpha \mapsto \omega^{\alpha}$
} 
(v) for every ordinal number $\gamma$ such that $\gamma \in \operatorname{succ} \alpha$ and $\gamma \neq \emptyset$ and $\gamma$ is a limit ordinal number holds $\varphi(\gamma)=\lim (\varphi\lceil\gamma)$.

The following propositions are true:

(41) $\varepsilon_{0}=\omega \uparrow \uparrow \omega$.

(42) $\varepsilon_{\operatorname{succ} \alpha}=\varepsilon_{\alpha} \uparrow \uparrow \omega$.

(43) Suppose $\beta \neq \emptyset$ and $\beta$ is a limit ordinal number. Let $\varphi$ be a sequence of ordinal numbers. If $\operatorname{dom} \varphi=\beta$ and for every $\gamma$ such that $\gamma \in \beta$ holds $\varphi(\gamma)=\varepsilon_{\gamma}$, then $\varepsilon_{\beta}=\lim \varphi$.

(44) If $\alpha \in \beta$, then $\varepsilon_{\alpha} \in \varepsilon_{\beta}$.

(45) For every sequence $\varphi$ of ordinal numbers such that for every $\gamma$ such that $\gamma \in \operatorname{dom} \varphi$ holds $\varphi(\gamma)=\varepsilon_{\gamma}$ holds $\varphi$ is increasing.

(46) Suppose $\beta \neq \emptyset$ and $\beta$ is a limit ordinal number. Let $\varphi$ be a sequence of ordinal numbers. If $\operatorname{dom} \varphi=\beta$ and for every $\gamma$ such that $\gamma \in \beta$ holds $\varphi(\gamma)=\varepsilon_{\gamma}$, then $\varepsilon_{\beta}=\bigcup \varphi$.

(47) If $\beta$ is a non empty limit ordinal number, then $x \in \varepsilon_{\beta}$ iff there exists $\gamma$ such that $\gamma \in \beta$ and $x \in \varepsilon_{\gamma}$.

$(48)^{4} \quad \alpha \subseteq \varepsilon_{\alpha}$.

(49) Let $X$ be a non empty set. Suppose that for every $x$ such that $x \in X$ holds $x$ is an epsilon number and there exists an epsilon number $e$ such that $x \in e$ and $e \in X$. Then $\bigcup X$ is an epsilon number.

(50) Let $X$ be a non empty set. Suppose that

(i) for every $x$ such that $x \in X$ holds $x$ is an epsilon number, and

(ii) for every $\alpha$ such that $\alpha \in X$ holds the first $\varepsilon$ greater than $\alpha \in X$.

Then $\bigcup X$ is an epsilon number.

Let us consider $\alpha$. Observe that $\varepsilon_{\alpha}$ is epsilon.

The following proposition is true

(51) If $\alpha$ is epsilon, then there exists $\beta$ such that $\alpha=\varepsilon_{\beta}$.

\section{Cantor Normal Form}

Let $A$ be a finite sequence of ordinal numbers. The functor $\sum A$ yielding an ordinal number is defined by the condition (Def. 8).

(Def. 8) There exists a sequence $f$ of ordinal numbers such that $\sum A=$ last $f$ and $\operatorname{dom} f=\operatorname{succ} \operatorname{dom} A$ and $f(0)=0$ and for every natural number $n$ such that $n \in \operatorname{dom} A$ holds $f(n+1)=f(n)+A(n)$.

One can prove the following propositions:

(52) $\sum \emptyset=0$.

\footnotetext{
${ }^{4}$ Of course not always $\alpha \in \varepsilon_{\alpha}$ because there are critical $\alpha$ 's such that $\alpha=\varepsilon_{\alpha}$
} 
(53) $\quad \sum\langle\alpha\rangle=\alpha$.

(54) For every finite sequence $A$ of ordinal numbers holds $\sum A^{\Upsilon}\langle\alpha\rangle=\sum A+\alpha$.

(55) For every finite sequence $A$ of ordinal numbers holds $\sum\langle\alpha\rangle^{\frown} A=\alpha+\sum A$.

(56) For every finite sequence $A$ of ordinal numbers holds $A(0) \subseteq \sum A$.

Let us consider $\alpha$. We say that $\alpha$ is Cantor component if and only if:

(Def. 9) There exist $\beta, n$ such that $0 \in n$ and $\alpha=n \cdot \omega^{\beta}$.

Let us note that every ordinal number which is Cantor component is also non empty.

Let us note that there exists an ordinal number which is Cantor component.

Let us consider $\alpha, \beta$. The functor $\beta$-exponent $(\alpha)$ yields an ordinal number and is defined by:

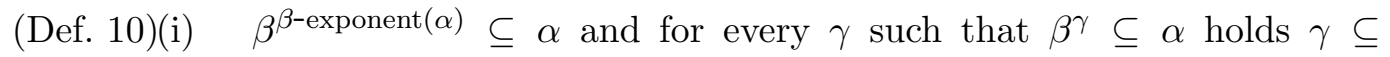
$\beta$-exponent $(\alpha)$ if $1 \in \beta$ and $0 \in \alpha$,

(ii) $\beta$-exponent $(\alpha)=0$, otherwise.

The following propositions are true:

(57) $\alpha \in \omega^{\operatorname{succ}(\omega-\operatorname{exponent}(\alpha))}$.

$(58)^{5}$ If $0 \in n$, then $\omega$-exponent $\left(n \cdot \omega^{\alpha}\right)=\alpha$.

(59) If $0 \in \alpha$ and $\gamma=\omega$-exponent $(\alpha)$, then $\alpha \div \omega^{\gamma} \in \omega$.

(60) If $0 \in \alpha$ and $\gamma=\omega$-exponent $(\alpha)$, then $0 \in \alpha \div \omega^{\gamma}$.

(61) If $0 \in \alpha$ and $\gamma=\omega$-exponent $(\alpha)$, then $\alpha \bmod \omega^{\gamma} \in \omega^{\gamma}$.

(62) If $0 \in \alpha$, then there exist $n, \beta$ such that $\alpha=n \cdot \omega^{\omega-\operatorname{exponent}(\alpha)}+\beta$ and $0 \in n$ and $\beta \in \omega^{\omega-\operatorname{exponent}(\alpha)}$.

(63) If $\omega$-exponent $(\beta) \in \omega$-exponent $(\alpha)$, then $\beta \in \alpha$.

Let $A$ be a sequence of ordinal numbers. We say that $A$ is Cantor normal form if and only if:

(Def. 11) For every $\alpha$ such that $\alpha \in \operatorname{dom} A$ holds $A(\alpha)$ is Cantor component and for all $\alpha, \beta$ such that $\alpha \in \beta$ and $\beta \in \operatorname{dom} A$ holds $\omega$-exponent $(A(\beta)) \in$ $\omega$-exponent $(A(\alpha))$.

Let us note that every sequence of ordinal numbers which is empty is also Cantor normal form and every sequence of ordinal numbers which is Cantor normal form is also decreasing and finite.

In the sequel $\mathcal{C}, \mathcal{B}$ are Cantor normal form sequences of ordinal numbers.

One can prove the following propositions:

(64) If $\sum \mathcal{C}=0$, then $\mathcal{C}=\emptyset$.

(65) If $0 \in n$, then $\left\langle n \cdot \omega^{\beta}\right\rangle$ is Cantor normal form.

Let us note that there exists a sequence of ordinal numbers which is non empty and Cantor normal form.

\footnotetext{
${ }^{5} \alpha$-exponent $(\beta)$ is the entier of the logarithm
} 
The following four propositions are true:

(66) Let $\mathcal{C}, \mathcal{B}$ be sequences of ordinal numbers. Suppose $\mathcal{C} \sim \mathcal{B}$ is Cantor normal form. Then $\mathcal{C}$ is Cantor normal form and $\mathcal{B}$ is Cantor normal form.

(67) If $\mathcal{C} \neq \emptyset$, then there exists a Cantor component ordinal number $\gamma$ and there exists $\mathcal{B}$ such that $\mathcal{C}=\langle\gamma\rangle^{\frown} \mathcal{B}$.

(68) Let $\mathcal{C}$ be a non empty Cantor normal form sequence of ordinal numbers and $\gamma$ be a Cantor component ordinal number. If $\omega$-exponent $(\mathcal{C}(0)) \in$ $\omega$-exponent $(\gamma)$, then $\langle\gamma\rangle \frown \mathcal{C}$ is Cantor normal form.

$(69)^{6}$ For every ordinal number $\alpha$ there exists a Cantor normal form sequence $\mathcal{C}$ of ordinal numbers such that $\alpha=\sum \mathcal{C}$.

\section{REFERENCES}

[1] Grzegorz Bancerek. The fundamental properties of natural numbers. Formalized Mathematics, 1(1):41-46, 1990.

[2] Grzegorz Bancerek. Increasing and continuous ordinal sequences. Formalized Mathematics, 1(4):711-714, 1990.

[3] Grzegorz Bancerek. König's theorem. Formalized Mathematics, 1(3):589-593, 1990.

[4] Grzegorz Bancerek. Ordinal arithmetics. Formalized Mathematics, 1(3):515-519, 1990.

[5] Grzegorz Bancerek. The ordinal numbers. Formalized Mathematics, 1(1):91-96, 1990.

[6] Grzegorz Bancerek. Sequences of ordinal numbers. Formalized Mathematics, 1(2):281290, 1990.

[7] Czesław Byliński. Functions and their basic properties. Formalized Mathematics, 1(1):5565, 1990.

[8] Agata Darmochwał. Finite sets. Formalized Mathematics, 1(1):165-167, 1990.

[9] Tetsuya Tsunetou, Grzegorz Bancerek, and Yatsuka Nakamura. Zero-based finite sequences. Formalized Mathematics, 9(4):825-829, 2001.

[10] Edmund Woronowicz. Relations and their basic properties. Formalized Mathematics, 1(1):73-83, 1990.

Received October 20, 2009

\footnotetext{
${ }^{6} \alpha=n_{1} \omega^{\beta_{1}}+n_{2} \omega^{\beta_{2}}+\cdots+n_{k} \omega^{\beta_{k}}$
} 